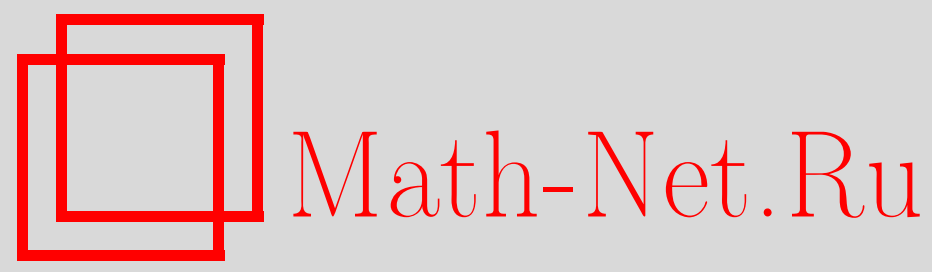

А. В. Абанин, И. С. Шабаршина, Характеризация порождающих идеалов в некоторых кольцах целых функций, $M a$ тем. заметки, 2003, том 74, выпуск 4, 483-493

DOI: https://doi.org/10.4213/mzm280

Использование Общероссийского математического портала Math-Net.Ru подразумевает, что вы прочитали и согласны с пользовательским соглашением http://www . mathnet.ru/rus/agreement

Параметры загрузки:

IP : 3.82 .47 .9

26 апреля 2023 г., 08:23:29

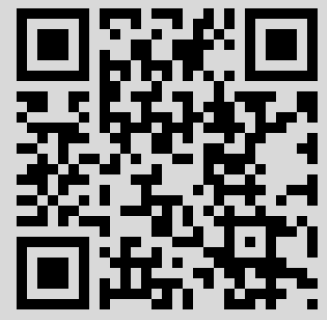




\title{
ХАРАКТЕРИЗАЦИЯ ПОРОЖДАЮЩИХ ИДЕАЛОВ В НЕКОТОРЫХ КОЛЬЦАХ ЦЕЛЫХ ФУНКЦИЙ
}

\section{А. В. Абанин, И. С. Шабаршина}

\begin{abstract}
Пусть $E$ - некоторое кольцо целых в $\mathbb{C}^{N}$ функций относительно операции поточечного умножения; $f_{1}, \ldots, f_{m}$ - фиксированный набор ненулевых элементов из $E$. Идеал $E\left(f_{1}, \ldots, f_{m}\right)$ в $E$ с образующими $f_{1}, \ldots, f_{m}$ называется порождающим, если $E\left(f_{1}, \ldots, f_{m}\right)=E$. Получена характеризация порождающих идеалов в кольцах целых в $\mathbb{C}^{N}$ функций, определяемых ростом их максимума модуля, в зависимости от распределения нулевых множеств их образующих. При дополнительном условии быстрого изменения весовых последовательностей, определяющих кольца, установлены критерии для порождающих идеалов, формулируемые в терминах $d(z):=\max _{1 \leqslant j \leqslant m} d_{j}(z)$, где $d_{j}(z)-$ расстояние от точки $z \in \mathbb{C}^{N}$ до нулевого множества $f_{j}, 1 \leqslant j \leqslant m$. Показано, что в кольцах целых функций, имеющих при фиксированном порядке конечньй или минимальный тип, подобная характеризация (т.е. характеризация через $d(z))$ невозможна.

Библиограффия: 10 названий.
\end{abstract}

1. Введение. Пусть $E$ - некоторое кольцо цельх в $\mathbb{C}^{N}$ функций относительно операции поточечного умножения; $f_{1}, \ldots, f_{m}$ - фиксированньй набор ненулевых элементов из $E$. Через $E\left(f_{1}, \ldots, f_{m}\right)$ обозначим идеал в кольце $E$, порожденньй функциями $f_{1}, \ldots, f_{m}$, т.е.

$$
E\left(f_{1}, \ldots, f_{m}\right):=\left\{f=\sum_{j=1}^{m} g_{j} f_{j}, \quad g_{j} \in E, \quad 1 \leqslant j \leqslant m\right\} .
$$

При этом $f_{1}, \ldots, f_{m}$ назьвают его образующими. Введенный идеал назьвается порождающим, если $E\left(f_{1}, \ldots, f_{m}\right)=E$. Начиная с работы Карлесона [1], относящейся к кольцу аналитических ограниченных в единичном круге функций, характеризация порождающих идеалов дается через оценки снизу для величины $\left|f_{1}(z)\right|+\cdots+\left|f_{m}(z)\right|$ [2]-[5]. В [6] наряду с традиционным получено описание таких идеалов в кольце $G$ всех целых в комплексной плоскости функций конечного порядка в терминах функции расстояния до нулей их образующих. Именно, пусть

$$
G:=\left\{\left.f \in H(\mathbb{C})|\exists \rho>0 \exists C>0: \ln | f(z)|\leqslant| z\right|^{\rho}+C \quad \forall z \in \mathbb{C}\right\},
$$

Работа выполнена при поддержке Российского фонда фундаментальных исследований, проект № 96-01-01041. 
$f_{j} \in[\infty, 0), f_{j}(z) \not \equiv 0,1 \leqslant j \leqslant m$. Положим

$$
d(z):=\max \left\{d \geqslant 0 \mid \exists j: 1 \leqslant j \leqslant m, f_{j}(\zeta) \neq 0 \text { при }|\zeta-z| \leqslant d\right\} .
$$

Тогда для того чтобы $G\left(f_{1}, \ldots, f_{m}\right)=G$, необходимо и достаточно, чтобы существовало такое $\rho>0$, что $d(z) \geqslant \rho^{-1} \exp \left(-\rho|z|^{\rho}\right)$ для любого $z \in \mathbb{C}$. Отметим, что в [6] аналогичная оценка приводится в несколько более сложной эквивалентной форме.

В настоящей работе получена характеризация порождающих идеалов в кольцах целых в $\mathbb{C}^{N}$ функций, определяемых ростом их максимума модуля, в зависимости от распределения нулевых множеств их образующих. В числе примеров пространств, укладывающихся в рамки сформулируемых ниже общих результатов, содержится как $G$, причем в многомерной ситуации, так и более "тонкие" кольца $[\rho, \infty),[\rho, 0]$ всех цельх в $\mathbb{C}^{N}$ функций, имеюших соответственно порядок не вьше $\rho$, конечный или минимальный тип при этом порядке. Кроме того, показано, что в этих более “тонких” кольцах полное описание порождающих идеалов в терминах функции $d(z)$ невозможно.

2. Основной результат. Пусть $h:[0, \infty) \rightarrow[0, \infty)$ - непрерывная возрастающая функция, для которой $\ln r=o(h(r))$ при $r \rightarrow \infty$ и $h(\exp r)$ - вьпуклая на $\mathbb{R}$ функция. Будем назьвать ее весовой или просто весом. Полагаем $h(z):=h(|z|)$ для $z \in \mathbb{C}^{N}$, где $|z|$ - обычная евклидова норма в $\mathbb{C}^{N}$. По $h$ определим весовое пространство целых в $\mathbb{C}^{N}$ функций

$$
E_{h}:=\left\{f \in H\left(\mathbb{C}^{N}\right)\left|\exists C=C_{f}>0: \ln \right| f(z) \mid \leqslant h(z)+C \forall z \in \mathbb{C}^{N}\right\} .
$$

В силу наложенных на $h$ условий $E_{h}$ содержит все полиномы. Для неубьвающей (невозрастающей) по $k$ последовательности $H=\left\{h_{k}\right\}_{k=1}^{\infty}$ весов рассмотрим пространство $E(H):=\bigcup_{k=1}^{\infty} E_{h_{k}}$ (соответственно $E(H):=\bigcap_{k=1}^{\infty} E_{h_{k}}$ ). Если нам необходимо отличить эти ситуации друг от друга, будем обозначать $H$ через $H_{\infty}$ в первом случае и через $H_{0}$ - во втором. В последующем предполагается и не оговаривается дополнительно, что $H_{\infty}$ (соответственно $H_{0}$ ) удовлетворяет такому ограничению:

$$
\begin{gathered}
\forall n \exists m \exists C>0: 2 h_{n}(2 r) \leqslant h_{m}(r)+C \forall r \geqslant 0 \\
\text { (соответственно } 2 h_{m}(2 r) \leqslant h_{n}(r)+C \quad \forall r \geqslant 0 \text { ). }
\end{gathered}
$$

Очевидно, что тогда $E(H)$ имеет структуру кольца относительно операции поточечного умножения и инвариантно относительно дифференцирования.

Пусть $g$ - целая в $\mathbb{C}^{N}$ отличная от тождественного нуля функция. При фиксированном $\zeta$, лежащем на единичной сфере $S_{1}:=\left\{z \in \mathbb{C}^{N}|| z \mid=1\right\}$, обозначим через $n_{g}(t ; \zeta)$ число нулей функции $g(\zeta u)$ в круге $\{u \in \mathbb{C}|| u \mid \leqslant t\}, t>0$. Положим

$$
\mathscr{N}_{g}(r):=\frac{1}{\sigma_{N}} \int_{S_{1}} d \sigma_{\zeta} \int_{0}^{r} n_{g}(t ; \zeta) d \ln t,
$$

где $d \sigma$ - элемент площади поверхности сферы $S_{1}$ в $\mathbb{C}^{N} \simeq \mathbb{R}^{2 N}$, а $\sigma_{N}=\int_{S_{1}} d \sigma$. Для характеризации массивности нулевого множества функции $g$ относительно произвольной фиксированной точки $z$ из $\mathbb{C}^{N}$ будем использовать величину $\mathscr{N}(g ; z ; r):=\mathscr{N}_{g(z+w)}(r)$, $r>0$. Ясно, что $\mathcal{N}(g ; z ; r)$ конечна тогда и только тогда, когда $g(z) \neq 0$. С целью упрощения записи полагаем $\mathcal{N}(g ; z)=\mathscr{N}(g ; z ;|z|)$ при $z \neq 0 ; \mathscr{N}(g ; 0)=0$, если $g(0) \neq 0$, и $\mathscr{N}(g ; 0)=\infty$, если $g(0)=0$.

Основной результат работы составляет 
Теорема 1. Следующие условия әквивалентны:

(i) идеал в кольие $E\left(H_{\infty}\right)$ (соответственно $\left.E\left(H_{0}\right)\right)$ с образующими $\left(f_{1}, \ldots, f_{m}\right)$ является порождаюшим;

(ii) существует $k \in \mathbb{N}$ (соответственно для любого $k \in \mathbb{N}$ ) существует $D$ $(=D(k))>0$ maкое, чmo

$$
\min _{1 \leqslant j \leqslant m} \mathscr{N}\left(f_{j} ; z\right) \leqslant h_{k}(z)+D \quad \forall z \in \mathbb{C}^{N} .
$$

ДокАЗАТЕЛЬСТво теоремы 1 разобьем на несколько этапов, проводя его по мере возможности одновременно для противоположных друг другу случаев $H=H_{\infty}$ и $H=H_{0}$.

1. Стандартным образом устанавливается, что (i) эквивалентно условию типа Карлесона

(iii) существует $s \in \mathbb{N}$ (соответственно для любого $s \in \mathbb{N}$ ) существует $c(=c(s))>0$ такое, что

$$
\left|f_{1}(z)\right|+\cdots+\left|f_{m}(z)\right| \geqslant c \exp \left(-h_{s}(z)\right) \quad \forall z \in \mathbb{C}^{N} .
$$

Именно, справедливость импликации (i) $\Rightarrow$ (iii) следует из того, что $f(z) \equiv 1 \in E(H)$, a обратной - (iii) $\Rightarrow(\mathrm{i})$, например, из результатов [5] (мы опускаем рутинные выкладки, связанные с применением упомянутых результатов к рассматриваемым кольцам).

Значит, доказательство теоремы 1 сводится к проверке равносильности условий (2) и (3) в согласованном относительно $H_{\infty}$ и $H_{0}$ виде. При этом, как (2), так и (3) влечет отсутствие общих нулей у $f_{1}, \ldots, f_{m}$ всюду в $\mathbb{C}^{N}$. Поэтому без ограничения общности можно предполагать, что $\left|f_{1}(z)\right|+\cdots+\left|f_{m}(z)\right| \neq 0$ в $\mathbb{C}^{N}$.

2. Для произвольной отличной от тождественного нуля целой в $\mathbb{C}^{N}$ функции $f$ и $z \in \mathbb{C}^{N}$ будем обозначать через $\mathfrak{M}(f ; z ; t)$ среднее функции $\ln |f|$ по сфере $\left\{w \in \mathbb{C}^{N} \mid\right.$ $|w-z|=t\}$, а через $\mathfrak{N}(f ; z ; t)$ - по шару $B(z ; t):=\left\{w \in \mathbb{C}^{N}|| w-z \mid \leqslant t\right\}, t>0$.

Пусть $f \in E_{h}, f(z) \not \equiv 0, h$-какой-либо вес. Тогда при некотором $C=C(h ; f)>0$

$$
\ln |f(z)| \leqslant h(z)+C \quad \forall z \in \mathbb{C}^{N} .
$$

Отсюда, при любом $z \in \mathbb{C}^{N}, z \neq 0$, выполнено

$$
\mathfrak{M}(f ; z ;|z|) \leqslant \ln \max \{|f(\zeta)|:|\zeta| \leqslant 2|z|\} \leqslant h(2 z)+C .
$$

Чтобы установить противоположную оценку, предположим сначала, что $f(z)$ не является тождественной постоянной (по-прежнему, $f \in E_{h}$ ). Тогда имеется $\xi \in \mathbb{C}^{N}$ c $|f(\xi)| \geqslant 1$. В силу плюрисубгармоничности $\ln |f(w)|$ в $\mathbb{C}^{N}$ при любом $z \in \mathbb{C}^{N}$ с $|z|>r_{0}=|\xi|$ имеем $\mathfrak{N}(f ; \xi ; 2|z|) \geqslant \ln |f(\xi)| \geqslant 0$. Отсюда, привлекая (4), получим при $|z|>r_{0}$

$$
\begin{gathered}
\int_{B(z ;|z|)} \ln |f(w)| d \omega_{w} \geqslant-\int_{B(\xi ; 2|z|) \backslash B(z ;|z|)} \ln |f(w)| d \omega_{w} \\
\geqslant-\omega_{N} 2^{2 N}|z|^{2 N}\left(h\left(2|z|+r_{0}\right)+C\right) \geqslant-\omega_{N} 2^{2 N}|z|^{2 N}(h(3 z)+C),
\end{gathered}
$$


где $d \omega$ - элемент объема в $\mathbb{C}^{N} \simeq \mathbb{R}^{2 N}, \omega_{N}=\int_{B(0 ; 1)} d \omega$. Поэтому

$$
\mathfrak{N}(f ; z ;|z|) \geqslant-2^{2 N}(h(3 z)+C), \quad z \in \mathbb{C}^{N}, \quad|z|>r_{0} .
$$

Поскольку всегда $\mathfrak{M}(f ; z ;|z|) \geqslant \mathfrak{N}(f ; z ;|z|)($ при $z \neq 0)$, то и

$$
\mathfrak{M}(f ; z ;|z|) \geqslant-2^{2 N}(h(3 z)+C), \quad z \in \mathbb{C}^{N}, \quad|z|>r_{0} .
$$

Очевидно, что если $f(z) \equiv c \neq 0$ в $\mathbb{C}^{N}$, то (6) вьполняется при $r_{0}=0$.

3. Допустим, что каждая из функций набора $f_{1}, \ldots, f_{m}$ принадлежит весовому пространству $E_{h}$. Тогда, использовав определение $E_{h}$ и оценки $(5),(6)$, получим, что имеются такие постоянные $C>0$ и $r_{0} \geqslant 0$, для которых

$$
\begin{array}{cl}
\ln \max _{1 \leqslant j \leqslant m}\left|f_{j}(z)\right| \leqslant h(z)+C & \forall z \in \mathbb{C}^{N} ; \\
\max _{1 \leqslant j \leqslant m} \mathfrak{M}\left(f_{j} ; z ;|z|\right) \leqslant h(2 z)+C & \forall z \in \mathbb{C}^{N} \backslash\{0\} ; \\
\min _{1 \leqslant j \leqslant m} \mathfrak{M}\left(f_{j} ; z ;|z|\right) \geqslant-2^{2 N}(h(3 z)+C), & z \in \mathbb{C}^{N}, \quad|z|>r_{0} .
\end{array}
$$

По формуле Йенсена (см., например, [7, с. 13]) для тех $j, 1 \leqslant j \leqslant m$, для которых $f_{j}(z) \neq 0$ (так как $f_{1}, \ldots, f_{m}$ не имеют общих нулей в $\mathbb{C}^{N}$, то для любого $z \in \mathbb{C}^{N}$ имеется хотя бы один такой индекс) вьполнено равенство

$$
\mathscr{N}\left(f_{j} ; z\right)+\ln \left|f_{j}(z)\right|=\mathfrak{M}\left(f_{j} ; z ;|z|\right)
$$

Отсюда с учетом (8) и (9) заключаем, что при $|z|>r_{0}$ вьполнена оценка

$$
-2^{2 N}(h(3 z)+C) \leqslant \min _{1 \leqslant j \leqslant m} \mathcal{N}\left(f_{j} ; z\right)+\ln \max _{1 \leqslant j \leqslant m}\left|f_{j}(z)\right| \leqslant h(2 z)+C .
$$

Предположим теперь, что при некотором $c>0$

$$
\left|f_{1}(z)\right|+\cdots+\left|f_{m}(z)\right| \geqslant c \exp (-h(z)) \quad \forall z \in \mathbb{C}^{N}
$$

Тогда $\ln \max _{1 \leqslant j \leqslant m}\left|f_{j}(z)\right| \geqslant-h(z)+\ln (c / m)$ для всех $z \in \mathbb{C}^{N}$, и из (10) следует

$$
\min _{1 \leqslant j \leqslant m} \mathcal{N}\left(f_{j} ; z\right) \leqslant h(2 z)+h(z)+C+\ln \frac{m}{c} \leqslant 2 h(2 z)+C_{1},
$$

где $C_{1}=C+\ln (m / c)$.

Обратно, если существует такое $D>0$, что

$$
\min _{1 \leqslant j \leqslant m} \mathcal{N}\left(f_{j} ; z\right) \leqslant h(z)+D \quad \forall z \in \mathbb{C}^{N} \backslash\{0\},
$$

то, снова привлекая (10), получим при $|z|>r_{0}$

$$
\ln \max _{1 \leqslant j \leqslant m}\left|f_{j}(z)\right| \geqslant-2^{2 N}(h(3 z)+C)-h(z)-D .
$$


Тем более, при тех же $z$ вьполняется неравенство

$$
\left|f_{1}(z)\right|+\cdots+\left|f_{m}(z)\right| \geqslant b \exp (-B h(3 z))
$$

где $b=\exp \left(-2^{2 N} C-D\right)>0, B=2^{2 N}+1$.

4. Чтобы закончить доказательство, остается заметить следующее. В случае $H=H_{\infty}$ имеются такие $l \in \mathbb{N}$ и $C>0$, что (7)-(10) выполнены при всех $h=h_{p}, p \geqslant l$. Если теперь при $s \in \mathbb{N}$ и $c>0$ справедливо (3) (совпадающее с (11) для $h=h_{s}$ ), то верно и (12) при $h=h_{n}$, где $n=\max \{l ; s\}$. Остается с помощью (1) выбрать такие $k \in \mathbb{N}$ и $A>0$, чтобы выполнялось неравенство $2 h_{n}(2 z) \leqslant h_{k}(z)+A$ при всех $z \in \mathbb{C}^{N}$. Тогда (12) влечет справедливость (2) с $D=C_{1}+A$.

Обратно, если вьполняется (2) (т.е. (13) при $h=h_{k}$ ), то, привлекая импликацию $(13) \Rightarrow(14)$, а затем (1), получим, что при некоторых $s \in \mathbb{N}$ и $c>0$ для $|z|>r_{0}$ верно (3). При этом, тот факт, что (3) справедливо для $z \in \mathbb{C}^{N} \mathrm{c}|z|>r_{0}$, не играет роли, так как изначально $f_{1}, \ldots, f_{m}$ не имеют общих нулей в $\mathbb{C}^{N}$ и за счет уменьшения $c>0$ легко добиться его справедливости всюду в $\mathbb{C}^{N}$.

Аналогично рассматривается случай $H=H_{0}$. Теорема доказана.

3. Порождающие идеалы, характеризуемые функцией расстояния до нулевых множеств. Пусть $f$ - целая в $\mathbb{C}^{N}$ функция, $V_{f}-$ ее нулевое множество и $d_{f}(z)$ - расстояние от точки $z \in \mathbb{C}^{N}$ до $V_{f}$. Для набора $f_{1}, \ldots, f_{m}$ цельх функций введем функцию $d(z):=\max _{1 \leqslant j \leqslant m} d_{f_{j}}(z), z \in \mathbb{C}^{N}$. Рассмотрим задачу о том, когда порождающие идеалы в кольцах типа $E(H)$ полностью характеризуются через $d(z)$. С целью упрощения записи будем говорить, что весовая последовательность $H_{\infty}$ (соответственно $H_{0}$ ) быстро возрастает (соответственно убывает), если для всех $k \in \mathbb{N}$ существуют $n \in \mathbb{N}, C>0$ такие, что

$$
\left.h_{k}^{2}(r) \leqslant h_{n}(r)+C \quad \text { (соответственно } h_{n}^{2}(r) \leqslant h_{k}(r)+C\right) \quad \forall r \geqslant 0 .
$$

Теорема 2. Для того чтобы идеал в кольие $E\left(H_{\infty}\right)$ (соответственно $E\left(H_{0}\right)$ ) с образуюшими $\left(f_{1}, \ldots, f_{m}\right)$ был порождающим, необходимо, а если $H_{\infty}$ (соответственно $H_{0}$ ) быстро возрастает (соответственно убывает), то и достаточно, чmoбbl

$$
\exists k \in \mathbb{N} \quad(\text { соответственно } \forall k \in \mathbb{N}) \quad \exists A>0: \ln \frac{1}{d(z)} \leqslant h_{k}(z)+A \quad \forall z \in \mathbb{C}^{N}
$$

ДокАЗАТЕЛЬСтво. По-прежнему, неограничивая общности, считаем, что $f_{1}, \ldots, f_{m}$ не имеют общих нулей в $\mathbb{C}^{N}$. Кроме того, сразу отбросим тривиальньй случай, когда хотя бы одна из функций рассматриваемого набора не обращается в нуль всюду в $\mathbb{C}^{N}$. Кстати, первое необходимо, а второе достаточно, как для того, чтобы идеал был порождающим, так и для выполнения (15).

Необходимость. Зафиксируем $z \in \mathbb{C}^{N} \backslash\{0\}$ и рассмотрим произвольную функцию $f_{j}$, для которой $f_{j}(z) \neq 0,1 \leqslant j \leqslant m$. По определению $d_{f_{j}}(z)=: d_{j}(z)$ имеется $\zeta$, лежащее на единичной сфере в $\mathbb{C}^{N}$, для которого функция переменной $u \in \mathbb{C} f_{j, z, \zeta}(u):=f_{j}(z+\zeta u)$ 
обращается в нуль хотя бы в одной точке окружности $\left\{u \in \mathbb{C}|| u \mid=d_{j}(z)\right\}$. Тогда, если $d_{j}(z) \leqslant|z| / 2$, то

$$
\mathscr{N}\left(f_{j, z, \zeta} ; \frac{|z|}{2}\right):=\int_{0}^{|z| / 2} n_{j, z, \zeta}(t) d t \geqslant \int_{d_{j}(z)}^{|z| / 2} d \ln t=\ln \frac{|z|}{2 d_{j}(z)}
$$

где $n_{j, z, \zeta}(t)$ - число нулей $f_{j, z, \zeta}(u)$ в круге $\{u \in \mathbb{C}|| u \mid \leqslant t\}$ с учетом их кратностей. Далее, по теореме 4.2.3 из [8] имеется постоянная $C>0$, зависящая только от размерности пространства $\mathbb{C}^{N}$, для которой $\mathscr{N}\left(f_{j, z}, \zeta ;|z| / 2\right) \leqslant C \mathscr{N}\left(f_{j} ; z\right)$. Поэтому при $d_{j}(z) \leqslant|z| / 2$ имеет место неравенство $\ln \left(|z| /\left(2 d_{j}(z)\right)\right) \leqslant C \mathscr{N}\left(f_{j} ; z\right)$. Отметим, что при $d_{j}(z)>|z| / 2$ оно вьполнено автоматически. Тогда $\ln (1 / d(z)) \leqslant C \min _{1 \leqslant j \leqslant m} \mathcal{N}\left(f_{j} ; z\right)$ при $|z| \geqslant 2$ и, следовательно, существует $D>0$ такое, что

$$
\ln \frac{1}{d(z)} \leqslant C \min _{1 \leqslant j \leqslant m} \mathscr{N}\left(f_{j} ; z\right)+D \quad \forall z \in \mathbb{C}^{N} .
$$

Остается воспользоваться теоремой 1 и условием (1), чтобы сделать заключение о необходимости (15) для того, чтобы идеал в кольце $E(H)$ с образующими $\left(f_{1}, \ldots, f_{m}\right)$ был порождающим.

Достаточность. Будем для простоты считать, что $\left|f_{j}(0)\right| \geqslant 1,1 \leqslant j \leqslant m$. Это не имеет принципиального значения, поскольку в противном случае можно поступить точно так же, как в теореме 1 , проводя рассуждения относительно $z_{j}$ с $\left|f_{j}\left(z_{j}\right)\right| \geqslant 1$ вместо нуля. Очевидно, $n_{j, z, \zeta}(t) \leqslant n_{j, 0, \zeta}(2|z|)$ при $|t| \leqslant|z|$. Воспользуемся известньп соотношением между числом нулей и максимумом модуля голоморфной функции одной переменной

$$
n_{j, 0, \zeta}(r) \leqslant \ln M_{f_{j}}(e r)-\ln \left|f_{j}(0)\right| \quad \forall r>0, \quad \forall \zeta \in S_{1} .
$$

Из него при $d_{j}(z) \leqslant|z|, z \neq 0$, вытекает

$$
\begin{aligned}
\mathscr{N}\left(f_{j} ; z\right) & =\frac{1}{\sigma_{N}} \int_{S_{1}} d \sigma_{\zeta} \int_{0}^{|z|} n_{j, z, \zeta}(t) d \ln t=\frac{1}{\sigma_{N}} \int_{S_{1}} d \sigma_{\zeta} \int_{d_{j}(z)}^{|z|} n_{j, z, \zeta}(t) d \ln t \\
& \leqslant \frac{1}{\sigma_{N}} \int_{S_{1}} d \sigma_{\zeta} \int_{d_{j}(z)}^{|z|} n_{j, 0, \zeta}(2|z|) d \ln t \leqslant \ln M_{f_{j}}(2 e|z|) \ln \frac{|z|}{d_{j}(z)},
\end{aligned}
$$

и, следовательно,

$$
\min _{1 \leqslant j \leqslant m} \mathcal{N}\left(f_{j} ; z\right) \leqslant\left(\max _{1 \leqslant j \leqslant m} \ln M_{f_{j}}(2 e|z|)\right) \cdot \ln \frac{|z|}{d(z)} .
$$

В последующем ограничимся случаем $H=H_{\infty}$ (случай $H=H_{0}$ рассматривается аналогично). По определению $H_{\infty}$ и условию (1) имеются $l \in \mathbb{N}$ и $C>0$ такие, что

$$
\max _{1 \leqslant j \leqslant m} \ln M_{f_{j}}(2 e|z|) \leqslant h_{l}(z)+C \quad \forall z \in \mathbb{C}^{N} .
$$

Тогда из условий (15) и (16) вытекает, что при $s=\max (k, l)$

$$
\min _{1 \leqslant j \leqslant m} \mathcal{N}\left(f_{j} ; z\right) \leqslant\left(h_{s}(z)+C\right)\left(h_{s}(z)+A+\ln |z|\right) .
$$


Воспользовавшись тем, что $\ln |z|=o\left(h_{s}(z)\right)$ при $z \rightarrow \infty$, быстрым возрастанием $H_{\infty}$ и еще раз условием (1), получим, что при некоторых $n \in \mathbb{N}$ и $D>0$ выполняется неравенство $\min _{1 \leqslant j \leqslant m} \mathscr{N}\left(f_{j} ; z\right) \leqslant h_{n}(z)+D$. Предыдушие рассуждения справедливы в предположении, что $d(z) \leqslant|z|, z \neq 0$. Остается заметить, что $\min _{1 \leqslant j \leqslant m} \mathcal{N}\left(f_{j} ; z\right)=0$ при $d(z)>|z|$ и последнее неравенство выполняется автоматически. Таким образом, справедливо $(2)$, и по теореме 1 идеал в кольце $E\left(H_{\infty}\right)$ с образующими $\left(f_{1}, \ldots, f_{m}\right)$ является порождаюшим. Теорема доказана.

Применим теорему 2 к пространствам $E_{\infty}$ и $E_{0}$ всех целых функций конечного и нулевого порядка соответственно:

$$
\begin{aligned}
E_{\infty} & :=\left\{\left.f \in H\left(\mathbb{C}^{N}\right)|\exists \rho>0 \exists C>0: \ln | f(z)|\leqslant C+| z\right|^{\rho}, \forall z \in \mathbb{C}^{N}\right\}, \\
E_{0} & :=\left\{\left.f \in H\left(\mathbb{C}^{N}\right)|\forall \rho>0 \exists C>0: \ln | f(z)|\leqslant C+| z\right|^{\rho}, \forall z \in \mathbb{C}^{N}\right\} .
\end{aligned}
$$

Очевидно, $E_{\infty}=E\left(H_{\infty}\right)$, где $H_{\infty}=\left\{|z|^{n}\right\}_{n=1}^{\infty}$ быстро возрастает, а $E_{0}=E\left(H_{0}\right)$, где $H_{0}=\left\{|z|^{1 / n}\right\}_{n=1}^{\infty}$ быстро убывает. Отметим, что $E_{\infty}$ совпадает с $G$, которое определялось во введении. Непосредственное применение теоремы 2 приводит к следующей характеризации порождающих идеалов в $E_{\infty}$ и $E_{0}$.

Теорема 3. Следующие условия эквивалентны:

(i) $E_{\infty}\left(f_{1}, \ldots, f_{m}\right)-$ порождающ, ий идеал;

(ii) найдется такое $\rho>0$, что в каждом шаре

$$
\left\{w \in \mathbb{C}^{N}|| w-z \mid \leqslant \rho^{-1} \exp \left(-|z|^{\rho}\right)\right\}, \quad z \in \mathbb{C}^{N} \text { произвольное }
$$

хотя бы одна из функиий набора $f_{1}, \ldots, f_{m}$ не обращается в нуль;

(iii) $f_{1}, \ldots, f_{m}$ не имеют общих нулей в $\mathbb{C}^{N} u$

$$
\limsup _{z \rightarrow \infty} \frac{\ln ^{+}(\ln 1 / d(z))}{\ln |z|}<\infty .
$$

Здесь, как обычно, $\ln ^{+} x=0$ при $x \leqslant 1$ и $\ln ^{+} x=\ln x$ при $x \geqslant 1$.

ТЕОРема 4. Следующие условия әквивалентны:

(i) $E_{0}\left(f_{1}, \ldots, f_{m}\right)-$ порождающий идеал;

(ii) для любого $\rho>0$ найдется $c>0$ такое, что при любом $z \in \mathbb{C}^{N}$ в шаре

$$
\left\{w \in \mathbb{C}^{N}|| w-z \mid \leqslant c \exp \left(-|z|^{\rho}\right)\right\}
$$

хотя бы одна из функиий набора $f_{1}, \ldots, f_{m}$ не обращается в нуль;

(iii) $f_{1}, \ldots, f_{m}$ не имеют общих нулей в $\mathbb{C}^{N} u$

$$
\lim _{z \rightarrow \infty} \frac{\ln ^{+}(\ln 1 / d(z))}{\ln |z|}=0
$$

Отметим, что теорема 3 - многомерное обобщение упомянутого во введении результата из [6]. Ясно, что перечень примеров можно было бы сушественно расширить. Например, под действие теоремы 2 подпадает $E\left(H_{\infty}\right)$, где $H_{\infty}=\left\{\ln ^{n}(1+|z|)\right\}_{n=2}^{\infty}$. Мы не будем на этом останавливаться, а рассмотрим кольца с более тонкой структурой и покажем, что для них характеризация порождающих идеалов в терминах функции расстояния $d(z)$ невозможна. Это будет, в частности, означать, что требование быстрого возрастания (убывания) существенно для справедливости теоремы 2. 
4. Пространства, определяемые уточненным порядком. Пусть $\rho(r)(\rightarrow \rho \in$ $(0, \infty))$ - уточненный порядок в смысле Валирона $[9], h(r):=r^{\rho(r)}$. Рассмотрим кольца целых в $\mathbb{C}^{N}$ функций, имеющих при этом уточненном порядке конечный и минимальный тип соответственно

$$
\begin{aligned}
{[\rho(r), \infty) } & :=\left\{f \in H\left(\mathbb{C}^{N}\right) \mid \limsup _{r \rightarrow \infty} \frac{1}{h(r)} \ln M_{f}(r)<\infty\right\} \\
{[\rho(r), 0] } & :=\left\{f \in H\left(\mathbb{C}^{N}\right) \mid \lim _{r \rightarrow \infty} \frac{1}{h(r)} \ln M_{f}(r)=0\right\} \cup\{0\} .
\end{aligned}
$$

Как известно, для $\rho(r)$ всегда существует эквивалентньй ему сильньй уточненньй порядок $\widetilde{\rho}(r)$, т.е. $r^{2} \widetilde{\rho}^{\prime \prime}(r) \ln r \rightarrow 0$ и $r^{\widetilde{\rho}(r)-\rho(r)} \rightarrow 1$ при $r \rightarrow \infty$. Например, подходит

$$
\widetilde{\rho}(r)=\rho+\frac{\ln l(r)}{\ln r}, \quad \text { где } l(r)=\frac{1}{r} \int_{1}^{r} t^{\rho(t)-\rho} d t .
$$

Тогда, очевидно, $[\widetilde{\rho}(r), \infty)=[\rho(r), \infty),[\widetilde{\rho}(r), 0]=[\rho(r), 0]$, и при этом функция $\widetilde{h}(r)=$ $r^{\widetilde{\rho}(r)}$ такова, что $\widetilde{h}(\exp x)$ вьпукла по крайней мере для больших $x$ [9]. Поэтому, не ограничивая общности, можно считать, что $\rho(r)$ - сильньй уточненньй порядок и $h(\exp x)$ выпукла на $\mathbb{R}$.

Легко видеть, что

$$
[\rho(r), \infty)=E\left(H_{\infty}^{\rho(r)}\right), \quad[\rho(r), 0]=E\left(H_{0}^{\rho(r)}\right)
$$

где

$$
H_{\infty}^{\rho(r)}:=\{k h(|z|)\}_{k=1}^{\infty}, \quad H_{0}^{\rho(r)}:=\left\{\frac{1}{k} h(|z|)\right\}_{k=1}^{\infty}
$$

Из свойств уточненного порядка [9] следует, что $H_{\infty}^{\rho(r)}$ и $H_{0}^{\rho(r)}$ удовлетворяют ограничению (1), и мы имеем возможность применить к рассматриваемым кольцам теорему 1 , непосредственная переформулировка условия (2) которой приводит к следующему результату.

Tеорема 5. Пусть $f_{1}, \ldots, f_{m}-$ набор функиий из кольца Е, не имеющих общих нулей в $\mathbb{C}^{N}$, где $E-[\rho(r), \infty)$ или $[\rho(r), 0]$. Для того чтобы идеал $E\left(f_{1}, \ldots, f_{m}\right)$ был порохдаюшим, необходимо и достаточно, чтобы для нулевых множеств его образующих имело место условие

$$
\begin{array}{rlll}
\limsup _{z \rightarrow \infty} & \frac{1}{h(|z|)} \min _{1 \leqslant j \leqslant m} \mathcal{N}\left(f_{j} ; z\right)<\infty & \text { в случае } & E=[\rho(r), \infty) \\
\lim _{z \rightarrow \infty} \frac{1}{h(|z|)} \min _{1 \leqslant j \leqslant m} \mathcal{N}\left(f_{j} ; z\right)=0 & \text { в cлучае } & E=[\rho(r), 0] .
\end{array}
$$

Приведем примеры, показывающие, что в отличие от $E_{\infty}$ и $E_{0}$ характеризация порождающих идеалов в кольцах $[\rho(r), \infty)$ и $[\rho(r), 0]$ в терминах функции $d(z)$ невозможна. Для этой цели достаточно ограничиться рассмотрением $N=1$ и $\rho(r) \equiv \rho>0$. 
ПРИМЕР 1. Пусть

$$
f(z)=\prod_{k=1}^{\infty}\left(1-\frac{z^{2}}{\lambda_{k}^{2}}\right)^{p_{k}}, \quad \text { где } \quad \lambda_{k}=k^{k / \rho}, \quad p_{k}=k^{k-1}, \quad k=1,2, \ldots
$$

Имеем

$$
\sum_{k=1}^{m} p_{k}=\sum_{k=1}^{m} k^{k-1} \leqslant(m-1)^{m-1}+m^{m-1}<2 m^{m-1} .
$$

Отсюда, в частности, следует, что $\lambda_{m}^{-\rho} \sum_{k=1}^{m} p_{k}<2 / m, m>1$, и, следовательно, $f \in$ $[\rho, 0]$. Положим

$$
f_{1}(z)=f(z), \quad f_{2}(z)=f(z-a), \quad \text { где } \quad a=\frac{1}{2} \min \left\{1,2^{2 / \rho}-1\right\} .
$$

Ясно, что $f_{1}$ и $f_{2}$ принадлежат кольцу $[\rho, 0]$ и, тем более, $[\rho, \infty)$. Так как

$$
\frac{\lambda_{k+1}}{\lambda_{k}}=\left(1+\frac{1}{k}\right)^{k / \rho}(k+1)^{1 / \rho} \geqslant 2^{2 / \rho}, \quad k \in \mathbb{N}
$$

и $\lambda_{1}=1$, то расстояние между любыми двумя соседними нулями функции $f_{1}$ (или $f_{2}$ ) не меньше $2 a$. Следовательно, в каждом круге $\{w \in \mathbb{C}|| z-w \mid<a / 2\}$ нет нулей хотя бы одной из функций $f_{1}$ или $f_{2}$. Таким образом, в рассматриваемом случае, когда образуюшими являются две функции $f_{1}$ и $f_{2}$, вьполнено

$$
\inf \{d(z): z \in \mathbb{C}\}=\frac{a}{2}>0
$$

Однако при $z_{m}=\lambda_{m}+a f_{2}\left(z_{m}\right)=0$, a

$$
\begin{aligned}
\mathcal{N}\left(f_{1} ; z_{m}\right) & =\int_{0}^{\left|z_{m}\right|} n_{f}\left(z_{m} ; t\right) d \ln t \geqslant \int_{a}^{\left|z_{m}\right|} p_{m} d \ln t=p_{m} \ln \frac{\left|z_{m}\right|}{a} \\
& \geqslant p_{m} \ln \lambda_{m}=\rho^{-1} m^{m} \ln m=\rho^{-1} \lambda_{m}^{\rho} \ln m
\end{aligned}
$$

$\left(n_{f}(z, t)\right.$ - число нулей $f$ в круге $\left.\{w \in \mathbb{C}|| w-z \mid \leqslant t\}\right)$.

Отсюда

$$
\limsup _{z \rightarrow \infty} \frac{1}{|z|^{\rho}} \min _{1 \leqslant j \leqslant 2} \mathscr{N}\left(f_{j} ; z\right) \geqslant \lim _{m \rightarrow \infty} \frac{1}{\left|z_{m}\right|^{\rho}} \mathscr{N}\left(f_{1} ; z_{m}\right)=+\infty
$$

и по теореме 5 идеалы $[\rho, \infty)\left(f_{1}, f_{2}\right)$ и $[\rho, 0]\left(f_{1}, f_{2}\right)$ не являются порождающими.

Отметим, что построенный пример опровергает результаты работы [10], в которой была предпринята попытка дать описание порождающих идеалов в кольце $[\rho, 0]$ посредством функции $d(z)$. Следующий пример показьвает, что подобная характеризация в принципе невозможна. 
ПРИМЕР 2. Пусть

$$
f_{1}(z)=\prod_{k=1}^{\infty}\left(1-\frac{z^{2}}{2^{2 k}}\right) .
$$

Эта функция имеет нулевой порядок. Оценим модуль этой функции снизу вне кружков

$$
\left\{z:\left|z \pm 2^{k}\right|<\frac{1}{2 k}\right\}, \quad k \in \mathbb{N},
$$

объединение которых обозначим через $U_{1}$. Пусть $z \in \mathbb{C} \backslash U_{1}$ и $m$ таково, что $2^{m} \leqslant$ $|z|<2^{m+1}$. Тогда

$$
\begin{aligned}
\ln \left|f_{1}(z)\right|= & \sum_{k=1}^{m-1} \ln \left|1-\frac{z^{2}}{2^{2 k}}\right|+\ln \left|1-\frac{z^{2}}{2^{2 m}}\right|+\ln \left|1-\frac{z^{2}}{2^{2(m+1)}}\right|+\sum_{k=m+2}^{\infty} \ln \left|1-\frac{z^{2}}{2^{2 k}}\right| \\
\geqslant & \sum_{k=1}^{m-1} \ln \left(\left|\frac{z}{2^{k}}\right|^{2}-1\right)+\ln \frac{\left|2^{m}-z\right|\left|2^{m}+z\right|}{2^{2 m}}+\ln \frac{\left|2^{m+1}-z\right|\left|2^{m+1}+z\right|}{2^{2 m+2}} \\
& +\sum_{k=m+2}^{\infty} \ln \left(1-\left|\frac{z}{2^{k}}\right|^{2}\right) \\
\geqslant & 2 \ln \frac{1}{2 m 2^{m}}+2 \ln \frac{1}{2(m+1) 2^{m+1}}+\sum_{k=1}^{\infty} \ln \left(1-\frac{1}{2^{2 k}}\right) \\
= & -2 \ln (4 m(m+1))-2(2 m+1) \ln 2+C,
\end{aligned}
$$

где

$$
C=\sum_{k=1}^{\infty} \ln \left(1-\frac{1}{2^{2 k}}\right)>-\infty
$$

Очевидно, что поведение последнего выражения определяется средним слагаемым, которое, в свою очередь, ведет себя как $-4 \ln |z|$. Поэтому при достаточно больших $|z|$, $z \in \mathbb{C} \backslash U_{1}$, выполняется оценка $\left|f_{1}(z)\right| \geqslant|z|^{-5}$.

Положим

$$
f_{2}(z)=\prod_{k=1}^{\infty}\left(1-\frac{z^{2}}{\mu_{k}^{2}}\right), \quad \text { где } \mu_{k}=2^{k}+\frac{1}{k} .
$$

Функция $f_{2}$ также имеет нулевой порядок. Оценки, аналогичные только что проведенным, показывают, что при больших $|z|, z \in \mathbb{C} \backslash U_{2}$, где

$$
U_{2}=\bigcup_{k=1}^{\infty}\left\{z \in \mathbb{C}:\left|z \pm\left(2^{k}+\frac{1}{k}\right)\right|<\frac{1}{2 k}\right\},
$$

выполнена оценка $\left|f_{2}(z)\right| \geqslant|z|^{-5}$. Поэтому найдется $R>0$ такой, что $\left|f_{1}(z)\right|+\left|f_{2}(z)\right| \geqslant$ $|z|^{-5}$ при $|z| \geqslant R$. Поскольку общих нулей у этих функций нет, то существует $c>0$ такой, что $\left|f_{1}(z)\right|+\left|f_{2}(z)\right| \geqslant c(1+|z|)^{-5}$ для всех $z \in \mathbb{C}$. Таким образом, для $f_{1}$ и $f_{2}$ выполнены условия типа Карлесона в любом из колец вида $[\rho, 0]$ или $[\rho, \infty), \rho>0$. То есть, при любом $\rho>0$ идеалы $[\rho, 0]\left(f_{1}, f_{2}\right)$ и $[\rho, \infty)\left(f_{1}, f_{2}\right)$ являются порождающими. 
$\mathrm{C}$ другой стороны, расстояние между ближайшими нулями $f_{1}$ и $f_{2}$, т.е. точками $2^{k}$ и $2^{k}+1 / k$ равно $1 / k$ и, значит,

$$
\liminf _{z \rightarrow \infty} d(z)=0
$$

Итак, условие (17) по примеру 1 не является достаточньм, а по примеру 2 необходимым для того, чтобы в кольцах вида $[\rho, 0]$ и $[\rho, \infty)$ соответствуюший идеал являлся порождающим.

\section{СПИСОК ЦИТИРОВАННОЙ ЛИТЕРАТУРЫ}

[1] Carleson L. Interpolation by bounded analytic functions and the corona problem // Ann. of Math. 1962. V. 76. №3. P. 547-559.

[2] Kelleher J. J., Taylor B. A. An application of the corona theorem to some rings of entire functions // Bull. Amer. Math. Soc. 1967. V. 73. № 2. P. 246-249.

[3] Hörmander L. Generators for some rings of analytic functions // Bull. Amer. Math. Soc. 1967. V. 73. №6. P. 943-949.

[4] Kelleher J. J., Taylor B. A. Closed ideals in locally convex algebras of analytic functions // J. Reine Angew. Math. 1972. V. 255. P. 190-209.

[5] Scoda H. Application des techniques $L^{2}$ a la théorie des idéaux d'une algèbre de fonctions holomorphes avec poids // Ann. Sci. École Norm. Sup. (4). 1972. V. 5. № 4. P. 545-579.

[6] Hennekemper W. Über Differentialideale im Ring der ganzen Funktionen endlicher Wachstumsordnung // Arch. Math. 1986. V. 46. P. 250-256.

[7] Ронкин Л.И. Целые функции // Итоги науки и техники. Современные проблемы математики. Фундаментальные направления. Т. 9. М.: ВИНИТИ, 1986. С. 5-36.

[8] Ронкин Л.И. Введение в теорию целых функций многих переменных. М.: Наука, 1971.

[9] Лелон П., Груман Л. Целые функции многих комплексных переменных. М.: Мир, 1989.

[10] Timofeev A. Ju. Die Differentialideale im Ring der ganzen Funktionen, die bei vorgegebener Ordnung Minimaltyp besitzen // Math. Nachr. 1990. V. 147. P. 89-94. 